\title{
Progressive Co-adaptation in Human-Machine Interaction
}

\author{
Paolo Gallina ${ }^{1}$, Nicola Bellotto ${ }^{2}$ and Massimiliano Di Luca ${ }^{3}$ \\ ${ }^{1}$ Department of Architecture and Engineering, University of Trieste, Via A. Valerio, 10, 34127, Trieste, Italy \\ ${ }^{2}$ Lincoln Centre for Autonomous Systems, School of Computer Science, University of Lincoln, Lincoln, U.K. \\ ${ }^{3}$ Centre for Computational Neuroscience and Cognitive Robotics \& School of Psychology, \\ University of Birmingham, Birmingham, U.K. \\ pgallina@units.it,nbellotto@lincoln.ac.uk,m.diluca@bham.ac.uk
}

\begin{abstract}
Keywords: Human-in-the-Loop, Usability, Teleoperation, Active Vision, Assistive Technology, Cyber-physical Systems.
Abstract: In this paper we discuss the concept of co-adaptation between a human operator and a machine interface and we summarize its application with emphasis on two different domains, teleoperation and assistive technology. The analysis of the literature reveals that only in a few cases the possibility of a temporal evolution of the co-adaptation parameters has been considered. In particular, it has been overlooked the role of time-related indexes that capture changes in motor and cognitive abilities of the human operator. We argue that for a more effective long-term co-adaptation process, the interface should be able to predict and adjust its parameters according to the evolution of human skills and performance. We thus propose a novel approach termed progressive co-adaptation, whereby human performance is continuously monitored and the system makes inferences about changes in the users' cognitive and motor skills. We illustrate the features of progressive co-adaptation in two possible applications, robotic telemanipulation and active vision for the visually impaired.
\end{abstract}

\section{INTRODUCTION}

In the field of Human-Computer Interaction (HCI) and, more generally, in the field of Human-Machine Interaction (HMI), the term co-adaptation refers to the process of adjustment of both the machine and the human operator during prolonged interaction. In other words, "both the human user and the machine should be able to adapt to the other through experiencing the interaction occurring between them" (Sawaragi, 2005). Designers of HMI applications can adopt several strategies for implementing adaptive changes in the HMI system with goals akin to co-adaptation: these approaches are defined humancentered and goal-oriented.

The aim of human-centered (or user-centered) coadaptation is to create a pleasant interface (Dixon, 2012) that maximizes usability. The design needs are shaped around the user skills and expectations. According to ISO 9241-210:2010 (Jokela et al., 2003), human-centered design is an "approach to systems design and development that aims to make interactive systems more usable by focusing on the use of the system and applying human factors/ergonomics and usability knowledge and techniques" (see Fig. 1).

Goal-oriented co-adaptation focuses instead on

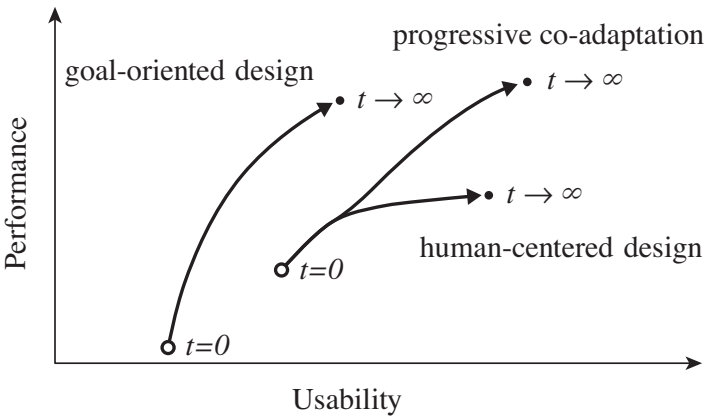

Figure 1: Performance and usability goals according to different HMI approaches. The separation between progressive co-adaptation and human-centered design should happen once performance is approaching a plateau.

designing a user interface that can be exploited at its maximum potential. This approach makes the assumption that the user is very skilled in performing the task. The drawback is that only well trained users can benefit from the adaptability of the system. A typical example of this approach is the use of joysticks to operate an excavator. Joysticks are widely used as human-machine interfaces in many applications of excavators, cranes, forklifts, electric-powered wheelchairs, and telemanipulated robots due to their reliability, ergonomy, and low cost. For some of these 
applications (e.g. excavators) the operator is required to undergo an extensive training as the mapping between the master device (joysticks) and the slave endeffector (shovel) can be counterintuitive. Another example of goal-oriented approach in a different domain is The vOICe, a mobile assistive device for the visually impaired that converts visual images to auditory signals (Ward and Meijer, 2010). In this case, given the richness of the information, the system requires long periods of training and user adaptation to the inherent cognitive load.

Performances and usability during interaction evolve in a different way depending on the selected approach, with human-centered design prioritizing usability increase and goal-oriented design giving more importance to performance increments (Fig. 1). The co-adaptation design approach aims to combine the advantages of human-centered design with the final performances achievable using the goal-oriented design by creating an interface that can adapt to the skills of the user assessed over time. It is adopted in all those applications where long-time training is required to make the interaction efficient and reliable.

Examples of co-adaptation design can be found in speech recognition software, gesture classifiers, and multimodal interfaces coupling human training and learning algorithms (Christoudias et al., 2006). Some form of co-adaptation is embedded in collaborative control strategies for robotic wheelchairs to determine the user's intention (e.g. desired destination) and adjust the control signals accordingly, adapting the level of assistance based on the affordances of the surroundings (Carlson and Demiris, 2012).

There are cases where co-adaptation is a requirement of the system. An example comes from the field of Brain-Computer Interfaces (BCI) applied to teleoperation applications. Electroencephalography (EEG) signals coming from the brain are collected, filtered, and coded in order to control a remote robot (Bi et al., 2013). For this types of applications, coadaptation is necessary because the system needs to learn how to associate desired commands with brain signals patterns while the participant is adapting to the novel task. The interface needs to be adaptive so to account for changes in cortical plasticity which modify brain connectivity leading to a different neural responses. This can be obtained in several ways. Bryan et al., for example, combined reinforcement learning and Bayesian inference (Bryan et al., 2013). Gürel and Mehring, instead, implemented an unsupervisedlearning decoder with a cost function derived from neuronal recording that allows on-line adjustment of its parameters (Gürel and Mehring, 2012).

In all these cases the interface should be able to dynamically modify its architecture at each time point, so to be able to optimise both long-term usability and performance. However, existing co-adaptation approaches are usually biased towards goal-oriented or human-centered interface improvements, which unavoidably lead to similar plateaus typical of nonadaptive solutions.

The main contributions of this paper is the definition and formalization of a new paradigm in HMI, named progressive co-adaptation. A progressive coadaptive interface is one that incorporates two functions: self-adaptation of the interface to the actual user skills, and simultaneous training of the user in order to increase long-term performances. The concept is discussed for two potential case scenarios, namely a robotic telemanipulation task and an active vision system for the visually impaired, which could both benefit from this novel approach.

\section{CO-ADAPTATION IN TASKS INVOLVING HUMAN MOTOR-LEARNING SKILLS}

In master-slave teleoperation implementations, the adaptation of the interface can take place for several reasons and at multiple levels. The control needs to account for various parameters, including time-delay (to increase performance and keep the system stable) (Chen et al., 2014), operator model uncertainties (related to human body impedance, not to cognitive or skill aspects) (Chan et al., 2014), and environment force uncertainties (Passenberg et al., 2010). Potential control problems are usually avoided by using classic control approaches (Hokayem and Spong, 2006) or with more advanced control techniques such as adaptive admittance control (Love and Book, 2004) or impact stabilization controllers (McAree and Daniel, 2000). In all these applications, the interface adapts immediately to the human and environment conditions to optimize the quality of the performed action in terms of transparency, stability, and reliability. Human adaptation of course still takes place, but it is not taken into account as a direct key-parameter to promote a modification of the interface.

If we indicate with $\chi$ the set of parameters that characterize the interface and that can be adjusted in order to adapt the interface to the human, then we can write the following:

$$
\chi=f(\Sigma)
$$

where $\Sigma$ represents the group of measurable parameters related to the aforementioned time-delay during 
telemanipulation, operator model uncertainties, environment force uncertainties, stability parameters, etc.

To elucidate this equation, we consider the mouse of a computer as an example. Such a control interface maps the two-dimensional motion on a surface to command a pointer on a display. For this interface, $\chi$ represents the speed parameter, the ratio between displacement of the cursor on the screen and displacement of the mouse on the surface.

Interface adaptation can take place at different levels and it can be prompted by parameters related to the operator skills. In master-slave teleoperation scenarios, the term Human Adaptive Mechatronics (HAM) indicates those interfaces that "are aimed to assisting the human according to his or her skill level by changing their own functions"(Harashima and Suzuki, 2010). For example, Furuta et al. proposed a haptic interface for operating a pendulumlike juggling slave mechanism (Furuta et al., 2011; Furuta, 2003) where a dedicated module provides a correction force controller to assist the human operator. Igarashi et al. proposed a graphical user interface, provided with effective alert functions, to reduce operator's misrecognition in a teleoperation task of a quadruped robot (Igarashi et al., 2005). Alert information is modulated depending on the human sensitivity to the features of the graphical user interface.

In this kind of applications it is necessary to perform a direct measure of the humans' skills (Suzuki et al., 2013) in terms of social abilities, planning, cognitive functions, dexterity, sensory-motor performance, or their combination. For this, models of human behavior models are required (Cui and Hua, 2013). Mavridis et al., for example, introduced a metric to evaluate operator's skills involved the teleoperation of a robot controlled by two joysticks. The metric considers kinematic parameters and is correlated to facial expression analysis (Mavridis et al., 2015b; Mavridis et al., 2015a). Suzuki et al. monitored the learning process involved in a bimanual teleoperation tasks (the tracking of two markers on a screen) by evaluating the tracking errors (Suzuki et al., 2008). Human skills can also be monitored by assistive technologies. In Hoey et al. (Hoey et al., 2010), for example, a vision-based system for automated handwashing assistance monitored the psychological state of a person with dementia to adapt accordingly. An alternative, indirect estimate of activity can be obtained by monitoring brain activity through EEG or Nearinfrared spectroscopy (Ishikuro et al., 2014).

To emphasize the key role of quantitative indexes in the interface adaptation process related to human skills, eq. 1 can be modified as follows:

$$
\chi=f(\Sigma, \Phi)
$$

where $\Phi$ represents the set of measurable parameters related to human skills. Note that in the humancentered design approach, the interface adjusts its parameters on-the-fly, as expressed by eq. 2. On the contrary, eq. 1 refers to the goal-oriented approach, where the interface does not account for the user skills. Back to the mouse example, $\Phi$ could be the average of the inverse of the error between the desired and the actual pointer position over a training section. Following eq. 2 , it is therefore necessary to introduce a metric that explicitly measures human skills over time.

\section{PROGRESSIVE CO-ADAPTATION}

All previously described adaptive techniques rely on the assumption that: a) there exists a relationship between the actual interface architecture $(\chi)$ and the human skills $(\Phi)$, and b) that the mapping between the two aspects is static and known a priori. In other words, adaptive techniques assume that there is a proper interface for each skill level of the user. However, as the inventor of the mouse Douglas Engelbart wisely stated: "if ease of use was the only valid criterion, people would stick to tricycles and never try bicycles". Indeed other factors, like the maximization of the overall performance, should be considered.

To achieve this, the relationship between the interface parameters $\chi$ and the human skills $\Phi$ can be defined as being dynamic rather than static:

$$
\chi=f(\Sigma, \Phi, t)
$$

where $t$ is the time, which becomes a key parameter in the design of the interface. For example, the mouse speed (i.e. $\chi$ parameter) could be linearly increased with time $(\chi=$ Costant $\times t)$. This simple strategy is meant to train non-skilled users over a long-time period. Note that, in the context of eq. 3 , the adaptation rate of the interface $\frac{d \chi}{d t}$ has to be properly calibrated, since it affects the stability of the system (Merel et al., 2013) as well as the final achievable performances of the human-machine interaction.

Another aspect that needs to be considered in a dynamic mapping interface is when the user is not able to improve the task performance (e.g. because of the limitation of the current interface parameters). This can be monitored by observing the term $\frac{d \Phi}{d t}$, which captures changes in the human skills $\Phi$. The interface can change to accommodate lacks of performance improvement, for example by linking the interface adaptation rate to $\frac{d \Phi}{d t}$. Therefore, the co-adaptation inter- 
face can be described as follows:

$$
\chi=f\left(\Sigma, \Phi, \frac{d \Phi}{d t}, t\right)
$$

Such equation is the general representation of the requirements of interfaces based on the notion of progressive co-adaptation, characterized by gradual but steady improvements. In practice, the separation between progressive co-adaptation and human-centered design should happen once the performance is approaching a plateau and $\frac{d \Phi}{d t}$ in eq. 4 decreases. In the following sections we will analyze two scenarios where the framework captured by this equation can be applied.

\section{APPLICATION \#1: TELEMANIPULATION}

An interface for teleoperation is made up of several "layers", each one with parameters that need to be modified over time for the co-adaptation to take place, even at the hardware level (Jin et al., 2014).

Operation performance depends not only on the mapping between slave DoFs and operator's DoFs (DoFs of the hands and/or arms involved in the remote operation), but also on several other geometric and control parameters, such as control gain (Huysmans et al., 2006) and stiffness (Oliver et al., 2006). To become the operator of heavy equipments, trainee require extensive training because of the counterintuitive and demanding cognitive mapping. Training simulators are often employed to reduce cost and time requirements. We believe that the parameters involved in teleoperation training can be modified over time according to the progressive co-adaptation paradigm in order to improve the effectiveness of the training procedures.

This approach can be applied to the domain of multi-robot teleoperation and, in particular, to the case of three $n$-DoFs independent serial robots teleoperated by a sole operator. To solve this issue we propose a control strategy based on a mixed control involving the Direct Rate control and the Resolved Position Control.

In the Direct Rate control manipulator there is a direct correspondence between each DoF of the master and each manipulator joint velocity. In this way, the master position is interpreted as a velocity command for the manipulator joint. Therefore the velocity can vary linearly with respect to the master position. Typically, this approach is used in excavators and cranes because the joystick position directly commands the hydraulic valve opening. In fact, there exists a linear relationship between the manipulator joint speed and the valve opening. In the Resolved Position Control, instead, the mapping is simply between each master DoF and the spatial DoFs of the manipulator.

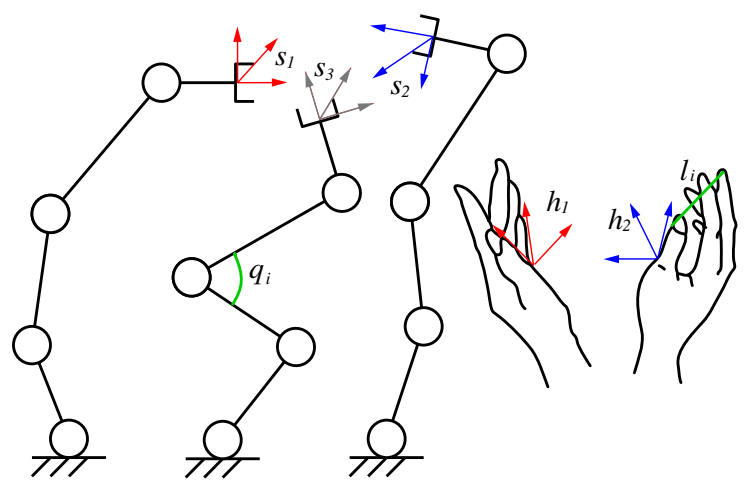

Figure 2: DoFs mapping between the 3 robots slave system and the two operator's hand poses.

The two approaches can be combined as shown in Fig. 2. A vision-based tracking device or a two glovebased master measures the positions of the operator's fingers. The two thumb poses $\mathbf{h}_{1}$ and $\mathbf{h}_{2}$ are used to control the end-effectors of two robots $\mathbf{s}_{1}$ and $\mathbf{s}_{2}$ according to a Resolved Position Control approach.

The third robot is controlled in a direct rate control mode by the other fingers. Let $\dot{q}_{i}$ be the command speed of each joint of the robot and $l_{i}$ the distance of a fingertip from the origin of the frame $\mathbf{h}_{1}\left(\right.$ or $\left.\mathbf{h}_{2}\right)$. The control role of each robot's joint is given then by the set of relations:

$$
\dot{q}_{i}=k_{i}\left(l_{i}-l_{i 0}\right)
$$

where $k_{i}$ and $l_{i 0}$ are parameters to be defined which characterize the human-robot interface. Therefore, in this case, the vector of the interface parameters is $\chi=\left\{k_{1}, \ldots, k_{n}, l_{10}, \ldots, l_{n 0}\right\}$. The latter can be adjusted adopting the proposed progressive co-adaptation approach.

For this application, we assume that the data transfer time-delays between the master device and the slave are negligible. Moreover, we assume that the slave system operates in a structured environment. Under these conditions, the parameter $\Sigma$ in eq. 4 is not relevant to the adjusting process and can be omitted. Instead, we need to define a metric to measure the operator's motor skills $\Phi$ over time. Let $\bar{e}$ be the error defined as the sum of the distance of the three end-effector poses $\mathbf{s}_{1}, \mathbf{s}_{2}$ and $\mathbf{s}_{3}$ from target poses imposed by the specific task $\mathbf{s}_{1_{\text {task }}}, \mathbf{s}_{2_{\text {task }}}$ and $\mathbf{s}_{3_{\text {task }}}$. The skills parameter $\Phi$ can be defined then as the average of the inverse error $1 / \bar{e}$ during each training section.

One possible progressive co-adaptation function could be implemented by increasing the parameters 
$k_{1}, \ldots k_{n}$ by a constant value every time $\frac{d \Phi}{d t}$ drops below a given threshold $\varepsilon$. In this way, as the user's skills tend to reach a plateau over time (additional training will not produce effects on motor skills), the interface parameters are adjusted in order to increase the overall performance of the human-robot interaction.

The co-adaptation process ends when no further operator' skill improvements are registered. This strategy would guarantee a stepwise training phase.

\section{APPLICATION \#2: ACTIVE VISION}

Another domain where co-adaptation can be applied is with mobile cyber-physical systems, in particular those addressing the fundamental problem of active vision with human-in-the-loop. The preliminary work in (Bellotto, 2013), for example, proposes a multimodal interface for handheld cameras devised to improve the navigation experience of the visually impaired. Such interface enables the user to point a smartphone's camera towards features of the environment facilitating for example navigation tasks like obstacle detection or place localization. In this scenario, co-adaptation between user and mobile device is particularly challenging due to the unpredictability of human motion and sensor uncertainty.

Active vision with human-in-the-loop is related to the classic problem of active perception, where the goal is to find models and control strategies that can facilitate the execution of a task (Bajcsy, 1988). In particular, the processes involved can be represented by a closed-loop system, in which the feedback from the mobile device and the vision algorithms are converted into "control signals" for the user to execute. The goal is to orient the camera towards particular objects or features in the environment, whose locations are used as reference for the system. Fig. 3 illustrates the concept. The input $r$ is the reference provided, for example, by some obstacle detection or a place localization algorithm, giving the direction of a visual target the camera should be pointing at. The error $e$ between the reference and the actual orientation $y$ of the camera is used by the controller $C$ to generate the control signal $u$.

Typical active vision systems are concerned with the optimal control of some electro-mechanical device that regulates the internal and/or external camera's parameters, like position, orientation, focal length, etc. (Rivlin and Rotstein, 2000). Active vision with human-in-the-loop, instead, tries to control the output of the whole human-camera subsystem, illustrated in Fig. 3 by block $H$ and $P$ respectively. Within

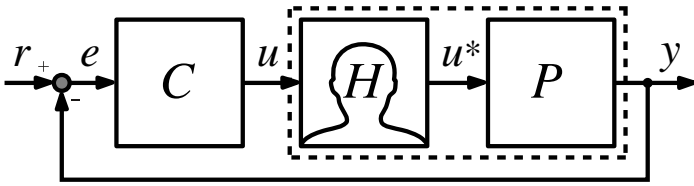

Figure 3: Feedback configuration with human-in-the-loop. The error $e$ is the difference between the input reference $r$ and system's output $y$. The control signal $u$ is generated by the controller $C$ to act on the human $H$. The latter moves the smartphone camera $P$ through another control signal $u^{*}$.

this subsystem, the control signal $u^{*}$ corresponds to the torque applied by the human to the handheld camera to change its direction and orientation.

In (Bellotto, 2013), a possible multimodal interface was proposed. The system's goal is to convey information that needs to be transmitted from the control algorithm $C$ to the user, i.e. to define a "signal" $u$ that, at least in most of the cases, can be interpreted correctly by the person within a reasonable time. An important aspect of this multimodal interface was the combination of vibrations, 3D sounds and vocal messages (on bone-conduction headphones) to instruct the user while pointing the smartphone's camera towards a visual target. The most efficient combination of the three modalities, as well as their individual tuning, can be achieved only by taking into account the long-term co-adaptation of the human-smartphone system.

The problem can be framed in the progressive coadaptation paradigm of eq. 4 by first defining the parameters of the smartphone's interface $\chi$. These include the position $p_{s}$ of the $3 \mathrm{D}$ sound source corresponding to the visual target, as well as the frequencies $\left\{f_{v}, f_{s}, f_{m}\right\}$ and the amplitudes $\left\{a_{v}, a_{s}, a_{m}\right\}$ of the vibrations, 3D sounds and vocal messages respectively. For example, $f_{m}$ would be the average number of vocal messages generated by the system within a fixed time interval, while $a_{m}$ would be the volume they are played at. Together, these parameters define the whole interface $\chi=\left\{p_{s}, f_{v}, f_{s}, f_{m}, a_{v}, a_{s}, a_{m}\right\}$.

Leaving aside the parameters $\Sigma$ related to timedelays etc., the next important element is the user "pointing" skill $\Phi$. One way to measure this is to compute the average error $\bar{e}$ of the control loop in Fig. 3 for a fixed time interval, then take its inverse (i.e. the smaller the error, the more skilled the user is).

As in the previous application, the interface parameters could be adjusted by increasing the value of one or more of them whenever $\frac{d \Phi}{d t}$ goes below a given threshold $\varepsilon$. For example, if during training the user does not improve in pointing the camera to the correct direction (i.e. $\frac{d \Phi}{d t}<\varepsilon$ ), the frequency $f_{m}$ of the vocal messages could increase to better assist him/her in the 
task. Note that in general there is not a linear relation between interface and skills parameters, and the map from one to the other can be much more complex than the examples provided here.

\section{CONCLUSIONS}

In this paper we proposed a general framework for the description of interface design for humanmachine interaction tasks involving motor-learning skills. In particular, two well-established approaches, the goal-oriented approach and human-centered approach, have been reviewed. Their advantages and drawbacks have been analyzed in terms of usability and performance, considering also their temporal evolution.

During the training phase of every long-term human-machine interaction, a process of neuroplasticity occurs: the user adapts to the interface and his/her motor skills improve. If, at the same time, the interface adjusts its characteristic parameters, the whole process can be referred to as a process of coadaptation. The analysis of time-adjusting interfaces coming from the literature reveals that the temporal evolution of the parameters related to co-adaptation (and in particular to the user's performance) has not been fully exploited. In particular, the role of timerelated indexes that capture changes in motor and cognitive abilities of the user has been overlooked.

A novel approach named progressive coadaptation is proposed to fill the gap. In this framework, human performance is continuously monitored and the system makes inferences about changes in the users' cognitive and motor skills. As argued in the paper, progressive co-adaptation attempts to combine the advantages derived from both the goal-oriented and human-centered approaches, while it mitigates their drawbacks. Indeed, for a more effective long-term co-adaptation process, the interface should be able to predict and adjust its parameters according to the evolution of human skills and performance (and not only to the actual ones).

To validate the proposed approach we plan to implement it for two practical applications of teleoperation and assistive technology, namely robotic telemanipulation and active vision for the visually impaired. For both cases, initial challenges have been described and potential solutions based on progressive co-adaptation have been discussed. We are reasonably confident that future research in this direction will highlight the advantages of the proposed approach.

\section{ACKNOWLEDGEMENTS}

We thank the EPSRC Network on Visual Image Interpretation in Humans and Machines for fostering this collaboration.

\section{REFERENCES}

Bajcsy, R. (1988). Active perception. Proc. of the IEEE, 76(8):966-1005.

Bellotto, N. (2013). A multimodal smartphone interface for active perception by visually impaired. In IEEE SMC Int. Workshop on Human-Machine Systems, Cyborgs and Enhancing Devices (HUMASCEND).

Bi, L., Fan, X.-A., and Liu, Y. (2013). Eeg-based braincontrolled mobile robots: A survey. IEEE Transactions on Human-Machine Systems, 43(2):161-176.

Bryan, M., Martin, S., Cheung, W., and Rao, R. (2013). Probabilistic co-adaptive brain-computer interfacing. Journal of Neural Engineering, 10(6).

Carlson, T. and Demiris, Y. (2012). Collaborative control for a robotic wheelchair: Evaluation of performance, attention, and workload. IEEE Trans. on Systems, Man, and Cybernetics - Part B, 42(3):876-888.

Chan, L., Naghdy, F., and Stirling, D. (2014). Application of adaptive controllers in teleoperation systems: A survey. IEEE Transactions on Human-Machine Systems, 44(3):337-352.

Chen, Z., Liang, B., Zhang, T., Zhang, B., and Song, H. (2014). An adaptive force reflection scheme for bilateral teleoperation. Robotica.

Christoudias, C., Saenko, K., Morency, L.-P., and Darrell, T. (2006). Co-adaptation of audio-visual speech and gesture classifiers. In ICMI'06: 8th International Conference on Multimodal Interfaces, pages 84-91.

Cui, Y. and Hua, J. (2013). Human behavior characteristics analysis in teleoperation system. Applied Mechanics and Materials, 373-375:163-166.

Dixon, J. (2012). Human Factors in Reliable Design, pages 137-155. John Wiley \& Sons, Inc.

Furuta, K. (2003). Control of pendulum: From super mechano-system to human adaptive mechatronics. In Proceedings of the IEEE Conference on Decision and Control, volume 2, pages 1498-1507.

Furuta, K., Kado, Y., Shiratori, S., and Suzuki, S. (2011). Assisting control for pendulum-like juggling in human adaptive mechatronics. Proceedings of the Institution of Mechanical Engineers. Part I: Journal of Systems and Control Engineering, 225(6):709-720.

Gürel, T. and Mehring, C. (2012). Unsupervised adaptation of brain-machine interface decoders. Frontiers in Neuroscience, 6(16).

Harashima, F. and Suzuki, S. (2010). State-of-the-art intelligent mechatronics in human-machine interaction. IEEE Industrial Electronics Magazine, 4(2):9-13.

Hoey, J., Poupart, P., von Bertoldi, A., Craig, T., Boutilier, C., and Mihailidis, A. (2010). Automated handwashing assistance for persons with dementia using 
video and a partially observable markov decision process. Computer Vision and Image Understanding, 114(5):503-519.

Hokayem, P. and Spong, M. (2006). Bilateral teleoperation: An historical survey. Automatica, 42(12):2035-2057.

Huysmans, M., de Looze, M., Hoozemans, M., van der Beek, A., and van Dieen, J. (2006). The effect of joystick handle size and gain at two levels of required precision on performance and physical load on crane operators. Ergonomics, 49(11):1021-1035.

Igarashi, H., Takeya, A., Kubo, Y., Suzuki, S., Harashima, F., and Kakikura, M. (2005). Human adaptive gui design for teleoperation system. In IECON Proceedings (Industrial Electronics Conference), pages 19731978.

Ishikuro, K., Urakawa, S., Takamoto, K., Ishikawa, A., Ono, T., and Nishijo, H. (2014). Cerebral functional imaging using near-infrared spectroscopy during repeated performances of motor rehabilitation tasks tested on healthy subjects. Frontiers in Human Neuroscience, 8(MAY).

Jin, X., Zhang, J., and Liu, Y. (2014). The ergonomics research of the joystick in excavator cab. Applied Mechanics and Materials, 494-495:128-131.

Jokela, T., Iivari, N., Matero, J., and Karukka, M. (2003). The standard of user-centered design and the standard definition of usability: Analyzing iso 13407 against iso 9241-11. In Proceedings of the Latin American Conference on Human-computer Interaction, CLIHC '03, pages 53-60.

Love, L. and Book, W. (2004). Force reflecting teleoperation with adaptive impedance control. IEEE Transactions on Systems, Man, and Cybernetics, Part B: Cybernetics, 34(1):159-165.

Mavridis, N., Pierris, G., Gallina, P., Papamitsiou, Z., Astaras, A., and Moustakas, N. (2015a). On the subjective difficulty of joystick-based robot arm teleoperation with auditory feedback. In Proc. of 8th IEEE GCC Conference and Exhibition.

Mavridis, N., Pierris, G., Gallina, P., Papamitsiou, Z., Astaras, A., and Moustakas, N. (2015b). Subjective difficulty and indicators of performance of joystick-based robot arm teleoperation with auditory feedback. In Proc. of IEEE International Conference on Robotics and Automation (ICRA).

McAree, P. and Daniel, R. (2000). Stabilizing impacts in force-reflecting teleoperation using distance-toimpact estimates. International Journal of Robotics Research, 19(4):349-364.

Merel, J., Fox, R., Jebara, T., and Paninski, L. (2013). A multi-agent control framework for co-adaptation in brain-computer interfaces. In Advances in Neural Information Processing Systems.

Oliver, M., Rogers, R., Rickards, J., Tingley, M., and Biden, E. (2006). Effect of stiffness and movement speed on selected dynamic torque characteristics of hydraulicactuation joystick controls for heavy vehicles. Ergonomics, 49(3):249-268.

Passenberg, C., Peer, A., and Buss, M. (2010). A survey of environment-, operator-, and task-adapted controllers for teleoperation systems. Mechatronics, 20(7):787801.

Rivlin, E. and Rotstein, H. (2000). Control of a camera for active vision: Foveal vision, smooth tracking and saccade. International Journal of Computer Vision, 39:8-96.

Sawaragi, T. (2005). Dynamical and complex behaviors in human-machine co-adaptive systems. In IFAC Proceedings Volumes (IFAC-PapersOnline), volume 16, pages 94-99.

Suzuki, S., Igarashi, H., Kobayashi, H., Yasuda, T., and Harashima, F. (2013). Human adaptive mechatronics and human-system modelling. International Journal of Advanced Robotic Systems, 10.

Suzuki, Y., Takase, H., Pan, Y., Ishikawa, J., and Furuta, K. (2008). Learning process of bimanual coordination. In 2008 International Conference on Control, Automation and Systems, ICCAS 2008, pages 28302835.

Ward, J. and Meijer, P. (2010). Visual experiences in the blind induced by an auditory sensory substitution device. Consciousness and Cognition, 19(1):492-500. 